\title{
Erratum to: Addressing End-of-Life Care in Cancer Patients through "Ubuntu": Lessons Learned from Rwanda in Global Health Perspective of Humanity
}

\author{
C. R. Ntizimira ${ }^{1}$ - S. Ngizwenayo $^{2}$ - E. L. Krakauer $^{3}$ - M. L. Dunne ${ }^{4} \cdot$ E. Esmaili $^{5}$
}

Published online: 21 February 2017

(C) Springer Science+Business Media New York 2017

\section{Erratum to: Current Obstetrics and Gynecology Reports DOI 10.1007/s13669-016-0186-7}

The permission statement for the use of Fig. 1 was incorrectly placed with the caption for Fig. 2. Figure 1 should be correctly listed as follows:

\section{WHO Analgesic Ladder}

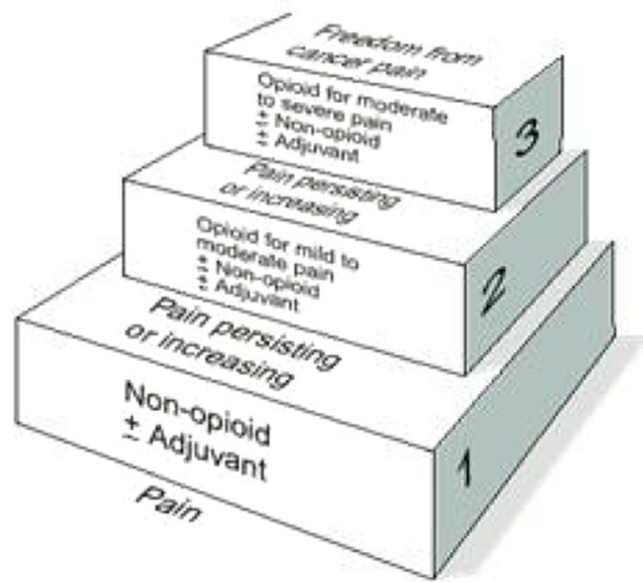

Fig. 1 The WHO analgesic ladder. Reprinted with permission from Cancer pain relief: with a guide to opioid availability, 2nd ed. World Health Organization, Evaluation of pain, page 15, WHO (2015). Retrieved from http://apps.who.int/iris/bitstream/10665/37896/1/ 9241544821.pdf. Accessed 10 September 2016

The online version of the original article can be found at http://dx.doi.org/ 10.1007/s13669-016-0186-7

C. R. Ntizimira

ntiheritier@gmail.com

1 AORTIC (African Organisation for Research and Training in Cancer), African Cancer Leaders Institute Fellow, KK 242 Street, Kicukiro District, Kigali, Rwanda
There were errors in the publishing of the "Introduction". The first paragraph of the introduction has been corrected to read as follows:

In his foreword in the Rwandan Ministry of Health's National Policy in Palliative Care, Minister Richard Sezibera states, "Over the last decade, Rwanda has made significant strides towards improved public health and achievement of the United Nations Millennium Development Goals. By extending the scope of available treatment to include palliative care, other domains such as maternal and child health or the combat against infectious diseases are reinforced... In the name of equal access to high quality health care, the Ministry of Health is devoted to integrating palliative care into the Rwandan health system. Our steadfast commitment is in light of a recent increase in the incidence of incurable diseases, chief among them HIV/AIDS and cancer." [1•]. Providing quality care remains the Ministry of Health's top priority, but this goal cannot be achieved without further developing human resources.

In the reference list, reference 12 was mistakenly included by the author, when it was not used in the article and should be removed from the list.

\footnotetext{
2 Kigali, Rwanda

3 Center for Palliative Care, Harvard Medical School, Boston, MA, USA

4 Vassar Brothers Medical Center, Poughkeepsie, NY, USA

5 Lincoln Community Health Center, Durham, NC, USA
} 
Consequently, references 13-20 should be renumbered to $12-19$. The citations $13-20$ in the text from pages 275-276 should be renumbered to 12-19.

Additionally, on page 276, reference 1 should have been reference 20 , which was incorrectly omitted by the author. The missing reference is as follows and should be listed as reference 20 in the reference list:

20. IntraHealth International. Rwanda: one of the first palliative care programs in Africa. 2011. http://www.intrahealth.org/page/rwanda-one-of-the-firstnational-palliative-care-policies-in-africa. Accessed 10 Sept 2016.

Consequently, references 20-23 should be renumbered to $21-24$. The reference numbers in the text on pages $276-277$ should re renumbered to $21-24$ as well. 